\title{
SAFETY OF ROUTINE MAGNETIC RESONANCE IMAGING IN CRITICALLY ILL PRETERM INFANTS AT 30 WEEKS GESTATIONAL AGE
}

\author{
A. Plaisier ${ }^{1,2}$, M. Feijen-Roon ${ }^{1,2}$, M.M.A. Raets ${ }^{1}$, C. van der Starre $^{1,3}$, P. Govaert ${ }^{1}$, M.H. Lequin ${ }^{2}$, A.M. \\ Heemskerk $^{1,2}$, J. Dudink ${ }^{1,2}$ \\ ${ }^{I}$ Neonatology, ${ }^{2}$ Radiology, ${ }^{3}$ Pediatric Intensive Care, Erasmus MC - Sophia, Rotterdam, The Netherlands
}

Background and aims: Preterm infants are at risk of brain injury with consequent neurodevelopmental impairment. As part of standard clinical care, we screen for possible brain injury by performing cerebral MRI at 30 weeks postmenstrual age. Our aim was to evaluate safety of MRI procedures in this population.

Methods: We retrospectively collected data regarding patients' safety and (avoidable) adverse events of 41 VLBW infants, whose MRI scans were routinely performed at 30 weeks of gestation. MRI procedures were carried out according to our multidisciplinary based guideline. This includes a time-out procedure and registration of vital parameters, adverse events and safety incidents related to the procedure.

Results: Mean gestational age (GA) and weight at image acquisition were 30.05 weeks (29.43-30.57) and 1112 grams (659-1580) respectively. No major adverse events occurred, but (preventable) incidents and minor adverse events were encountered: MRI procedure was interrupted in 3 infants $(7.3 \%)$ because of respiratory instability. Minor increased respiratory support within 24 hours after the MR procedure was necessary in 10 infants $(24.4 \%)$. Hypothermia (temperature $\left.<36^{\circ} \mathrm{C}\right)$ occurred in 7 infants $(17.1 \%)$. There was no significant correlation between temperature and GA at birth, weight at image acquisition, mode of respiratory support or hemodynamic instability.

Conclusions: Although MRI scanning at 30 weeks gestational age is relatively safe for preterm infants, adverse events are common and should not be underestimated. A tailored guideline for MRI procedures in preterm infants including registration of patient data, time-out procedures and incident review is essential and the guideline should continuously be re-evaluated. 